\title{
Neurophysiology of robot-mediated training and therapy: a perspective for future use in clinical populations
}

\author{
Duncan L. Turner ${ }^{1,2}{ }^{*}$, Ander Ramos-Murguialday ${ }^{3,4}$, Niels Birbaumer ${ }^{3,5}$, Ulrich Hoffmann ${ }^{4}$ and Andreas Luft ${ }^{6}$ \\ ${ }^{1}$ Neurorehabilitation Unit, University of East London, London, UK \\ 2 Lewin Stroke Rehabilitation Unit, Department of Clinical Neurosciences, Cambridge University NHS Foundation Trust, Cambridge, UK \\ ${ }_{3}^{3}$ Institute of Medical Psychology and Behavioral Neurobiology, MEG Center, University of Tubingen, Tubingen, Germany \\ ${ }^{4}$ Health Division, Tecnalia Research \& Innovation, San Sebastian, Spain \\ ${ }^{5}$ Ospedale San Camillo, Istituto di Ricovero e Cura a Carattere Scientifico, Venezia Lido, Italy \\ ${ }^{6}$ Clinical Neurorehabilitation, Department of Neurology, University of Zurich, Zurich, Switzerland
}

Edited by:

Cuntai Guan, Institute for Infocomm Research, Singapore

\section{Reviewed by:}

Kenji Kansaku, Research Institute of

National Rehabilitation Center for

Persons with Disabilities, Japan

Roberto Colombo, Fondazione

Salvatore Maugeri, Italy

*Correspondence:

Duncan L. Turner, Neurorehabilitation Unit, School of Health, Sport and

Bioscience, University of East

London, Stratford, London E15 4LZ,

UK

e-mail:d.I.turner@uel.ac.uk
The recovery of functional movements following injury to the central nervous system (CNS) is multifaceted and is accompanied by processes occurring in the injured and non-injured hemispheres of the brain or above/below a spinal cord lesion. The changes in the CNS are the consequence of functional and structural processes collectively termed neuroplasticity and these may occur spontaneously and/or be induced by movement practice. The neurophysiological mechanisms underlying such brain plasticity may take different forms in different types of injury, for example stroke vs. spinal cord injury (SCI). Recovery of movement can be enhanced by intensive, repetitive, variable, and rewarding motor practice. To this end, robots that enable or facilitate repetitive movements have been developed to assist recovery and rehabilitation. Here, we suggest that some elements of robot-mediated training such as assistance and perturbation may have the potential to enhance neuroplasticity. Together the elemental components for developing integrated robot-mediated training protocols may form part of a neurorehabilitation framework alongside those methods already employed by therapists. Robots could thus open up a wider choice of options for delivering movement rehabilitation grounded on the principles underpinning neuroplasticity in the human CNS.

Keywords: motor cortex, spinal cord, rehabilitation, motor learning, motor adaptation

\section{INTRODUCTION}

Stroke or spinal cord injury (SCI) often leaves an individual with persistent functional movement deficits that impact on independent living and quality of life, whilst putting an enormous healthcare and macro-economic burden on societies (1). Such sudden onset injury to the central nervous system (CNS) was long considered immune to treatment. However, in the last three decades a paradigm shift has occurred whereby a better understanding of recovery has highlighted the potential for re-organization of neural circuits that remain intact after stroke or SCI (2-4). Recovery involves several regions of the CNS and can spontaneously occur after stroke or incomplete SCI, that is, in the absence of specific training.

Several potential therapies may assist or guide this spontaneous recovery and include constrained induced movement therapy (CIMT), robot-mediated therapy, pharmacological treatments (e.g., selective serotonin re-uptake inhibitors), brain-machine interfaces (BMIs), goal oriented physiotherapy, epidural spinal stimulation, non-invasive cortical stimulation, electromechanicalmediated therapy, and combinations thereof (5-13). Taking two examples, both CIMT (the EXCITE trial) and robotmediated therapy (the VA robot trial) have been demonstrated to induce better clinical outcomes than usual care following stroke $(9,13)$.

\section{NEUROPLASTICITY IN THE HUMAN BRAIN}

Underlying many existing motor therapies is the central tenet that repetitive, progressive, and engaging practice using the affected limb induces plastic changes in neural networks subserving motor control and learning. The changes could be both functional and structural-anatomical and the neurophysiological processes by which these changes might occur, have been collectively termed as neuroplasticity $(2,3,14,15)$.

Neuroplasticity occurs at synapses and involves molecular changes in cell signaling pathways and neurotransmission; both dendritic and axonal plasticity can occur in healthy conditions and also after damage to the CNS (16-18). There are spike timedependent changes in neuronal synaptic strength that can be demonstrated in response to high-frequency stimulation in in vitro and in vivo animal studies and which contribute to changes in neurophysiology such as increased or decreased evoked postsynaptic potentials (EPSPs) that can persist for long periods [i.e., long-term potentiation or depression; LTP and LTD (18)]. Since the pioneering studies of the 1960s and 1970s and subsequent rapid consolidation of understanding of mechanisms underpinning LTP/LTD, induced changes in synaptic strength have also been directly demonstrated in vitro in human tissue surgically excised from either the hippocampus or neocortical temporal lobe (19, 20). More recent studies in humans, have demonstrated analogous 
changes in cortical excitability following high-frequency sensory stimulation (19). Paired associative conditioning stimulation paradigms (PAS) such as non-invasive peripheral nerve stimulation paired with non-invasive transcranial magnetic stimulation (PNS and TMS respectively) as well as non-invasive weak transcranial direct current stimulation (tDCS) can also induce LTP/LTD-like changes in motor cortical excitability and are mediated by complex neurotransmitter and neuromodulatory systems in a similar manner to the original in vitro animal studies (21). Thus the human brain has the capacity for neuroplastic adaptation to changing environmental conditions.

The next translational step to make in favor of human neuroplasticity is to demonstrate that changes in synaptic strength resulting from these basic molecular, cellular, and neurophysiological phenomena can lead to re-organization of neural connectivity at the local small world network level, across the cerebral hemispheres, along the spinal cord segments and ultimately could occur across the whole CNS system. An approach to this is to combine neuroimaging of the whole brain (e.g., functional magnetic resonance imaging; fMRI) and site-specific non-invasive brain stimulation (e.g., tDCS on motor cortex). For example, applying unilateral anodal tDCS to motor cortex reduces resting interhemispheric cortical and contralateral intra-cortical functional connectivity (22), but increases ipsilateral motor-premotor, motor-parietal cortical functional connectivity as well as corticostriato-thalamic functional connectivity $(23,24)$. Thus, the adult human CNS appears to have the capacity to adapt to artificial (e.g., tDCS) and more natural stimulation (e.g., visual or auditory stimuli), both in terms of cell-based neurophysiology and at neural network-based levels, thereby demonstrating an innate capacity to undergo neuroplasticity.

\section{NEUROPLASTICITY IN THE CLINIC}

Several recent reviews cover general aspects of rehabilitation following stroke and SCI and the potential role of neuroplasticity in recovery processes (25-33). Here we specifically focus on the potential of robot-mediated therapy to induce neuroplasticity as evidenced by some or all of the basic phenomena highlighted. There is a growing evidence-base for neuroplasticity to occur in healthy subjects when they engage with robot devices in studies of motor learning (Figure 1).

Whether these learning mechanisms demonstrated in health also occur during rehabilitation employing robot devices for neurological recovery is not fully established in the literature, we therefore highlight some recommendations for future research rather than a meta-analysis of available evidence. We will highlight points of caution where we translate evidence for examples of robot-mediated neuroplasticity in learning in healthy adults to those individuals with CNS injury (34).

\section{NEUROPLASTICITY AND ROBOT-MEDIATED LEARNING}

This perspective focuses on four elements of robot-mediated learning with respect to their potential to induce neuroplasticity in clinical populations. Evidence from studies on healthy subjects and then on neurological populations will be described and a summary on potential future research areas put forward for each element.

\section{ELEMENT 1: ROBOTIC ASSISTANCE}

Assistance by a robot involves the device providing a haptic interaction $^{1}$ and there is a growing range of control strategies associated with it [(35) for a comprehensive review]. For example, this could incorporate a "haptic tunnel" for the movement path (36) in the form of forces provided by actuators to reach a movement target when the patient is not able to perform the desired range of motion $(37,38)$ or in the form of correct movements performed by a robot not in contact with the patient [i.e., the robot has a coaching role; $(35,39)]$. Strategies correlating contingent proprioceptive and/or other sensory inputs to motor outputs also might be important for inducing neurophysiological changes $(18,40)$.

\section{EVIDENCE FOR NEUROPLASTICITY: UPPER LIMB}

Recent work has demonstrated that robot-assisted wrist movements or hand grip in healthy subjects are accompanied by different frequency-dependent power changes in the electroencephalogram (EEG) in neural cortical circuits compared to voluntary wrist movement or hand grip $(41,42)$. Furthermore, assistive haptic feedback during a visuomotor tracking task induces region-specific changes in frequency-dependent power compared to tracking with no haptic feedback. Interestingly, there are also increases in functional connectivity (coherence) between cortical regions involved in the motor task only when assistive haptic feedback is present (43). Robot-assisted unilateral wrist movement modulates contralateral alpha and beta frequency power (desynchronization) in cortical areas that are also involved during voluntary wrist movements (41). Further, the movement-evoked potentials of voluntary and assisted (non-robotic in this case) finger movements are at similar times ( 35 vs. $36 \mathrm{~ms}$ respectively) after movement onset and are in the same current source locations (44). Substantial overlap of neural activity representation is also demonstrated for elbow flexion/extension in voluntary and torque-motor (i.e., similar to robotic) driven conditions (45). Thus (active) voluntary and (quasi-passive) robot-assisted motor tasks activate similar brain regions.

However, neuroplasticity per se is considered to be underpinned by progressive, challenging motor skill learning rather than merely repetitive motor tasks. Active voluntary motor skill learning with the wrist leads to more prominent increases in (i) activity in contralateral primary motor cortex, (ii) motor excitability recruitment curves, and (iii) intracortical facilitation compared to passive (torque-motor assisted) motor skill learning (46). The greater changes in motor excitability in active voluntary vs. passive motor skill learning have also been repeated for ankle flexion/extension in visuomotor tracking (47). These findings suggest that robot-assisted motor skill learning may not necessarily be as influential as voluntary motor skill learning in inducing neuroplasticity. Encouragingly however, there is evidence to suggest that re-organization of brain networks can occur after robot-assisted therapy in stroke patients both

\footnotetext{
${ }^{1}$ Relating to the sense of touch, in particular relating to the perception and manipulation of objects using the senses of touch and proprioception: hapticfeedback devices create the illusion of substance and force within the virtual world. Oxford Dictionaries, Oxford University Press, Oxford, England 2012.
} 


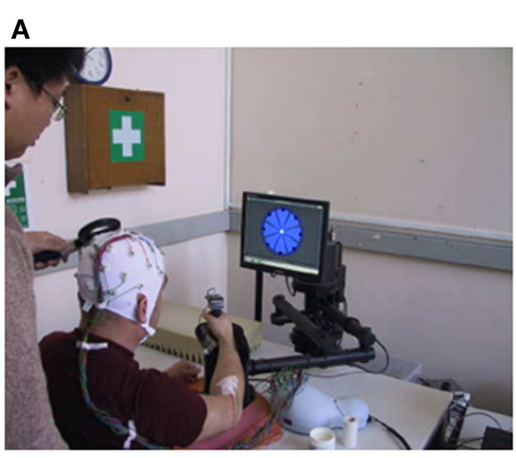

B

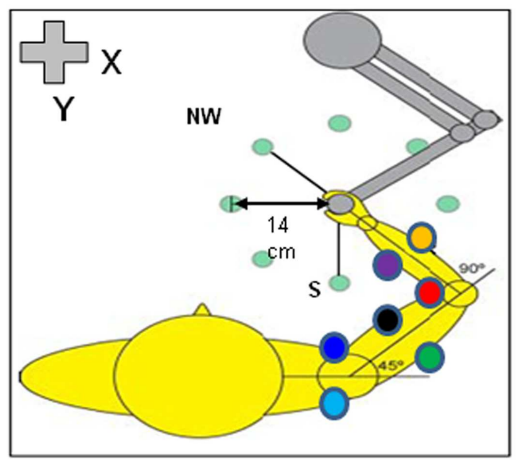

FIGURE 1 | An upper limb end-effector robotic device can be used to monitor cortical and neuromuscular responses with TMS, EEG, and EMG (electrodes placed on multiple shoulder, arm, forearm muscles) during performance of reaching movements in different directions in the $\boldsymbol{x}-\boldsymbol{y}$ axis $(\mathbf{A}, \mathbf{B})$. The motors can be switched off to measure "free" movements or switched on to induce force fields (perturbation or resistance). Other adjunct methods of brain stimulation can be used during robot-mediated reaching movements such as tDCS (C); different
C
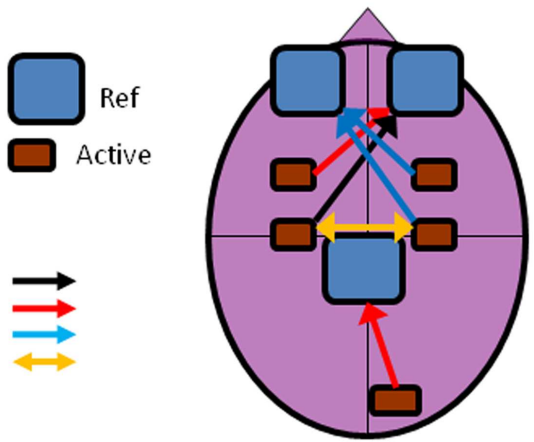

D

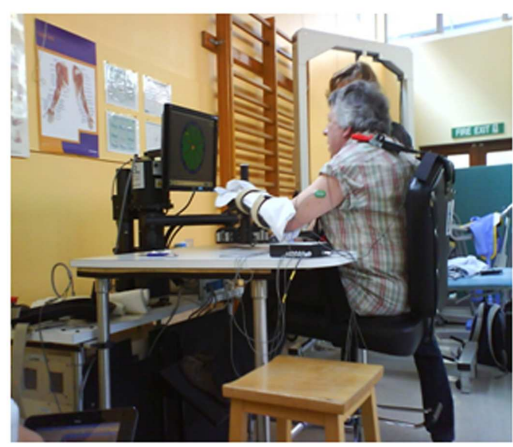

types of tDCS include: unilateral anodal motor cortex - black arrow, unilateral anodal premotor or visual cortex - red arrows, unilateral cathodal stimulation - blue arrows or directional stimulation - yellow arrow; Ref, reference electrode, Active, active electrode). The robotic device can be used to assist acute stroke patients in reaching motor practice in therapy or be programed to perturb motor performance to measure patient kinematic performance and muscle responses in different tasks such as position holding [(D); see also Figure 4]. in terms of regional activation (48) and interhemispheric and intrahemispheric functional connectivity (49).

\section{EVIDENCE FOR NEUROPLASTICITY: LOWER LIMB}

Stroke and SCI can impede the ability to walk significantly and reduce independence in living. Surprisingly therefore, there is little knowledge of the neural mechanisms underlying lower limb functional recovery; even less is known about the impact of walking rehabilitation on the neural aspects of recovery and virtually nothing is known about the impact of robot-assisted therapy of the lower limb on neuroplasticity.

Cortical control of locomotion involves a complex interplay of supraspinal circuits, spinal interneurons, and spinal reflexes. Either spinal or supraspinal (stroke) injury could bring about re-organization of all levels of the neuroaxis. A full review of the neural correlates of locomotor control in clinical populations is beyond this perspective [see Ref. $(25,50,51)]$. Nevertheless some common aspects can be demonstrated whereby unilateral hemispheric stroke effects functional (measured with TMS) and structural (measured with diffusion tensor MRI) corticospinal tract integrity and this is proportional to walking impairment (52). On the other hand, corticospinal tract integrity above an incomplete cervical spinal lesion is also reduced in terms of spinal cord area, smaller white matter volumes in pyramids and left cerebellar peduncle and smaller gray matter volume in the leg area of the motor cortex - importantly, clinical impairment was correlated with some of these functional-structural measures (53). Whilst the specific changes may be different between stroke and SCI, both types of injuries can be associated with brain re-organization.

Can these re-organizations of brain function and structure be "tuned" or enhanced by motor training? There is strikingly little information available to answer this especially in the early stages of recovery (54). Longitudinal imaging studies have documented an increase in neural activation in midbrain and cerebellum following extended aerobic walking training in chronic stroke patients [i.e., along with the post-stroke re-organization detailed earlier; (55)]. Walking velocity was correlated with midbrain and cerebellar activation, suggestive of neuroplasticity underpinning clinical improvement. Both cortical and subcortical regions appear to be involved in walking training rehabilitation intervention in chronic stroke $(56,57)$. The balance between cortical and subcortical neuroplasticity in these two similar study paradigms may have been due to whether proximal (55) or distal leg muscle function was tested during fMRI (56). Robot-assisted, body-weight supported walking training on a treadmill resulted in greater sensorimotor 
and cerebellar activation following a prolonged intervention after incomplete SCI, (58) and greater corticomotor responses to transcranial magnetic stimulation of the leg cortical representation during stroke rehabilitation (59). Finally, robot-assisted treadmill walking training (with concomitant cognitive and imagery training) increased sensorimotor neural activation and functional connectivity in a case study of adult traumatic brain injury (60).

The overall evidence that healthy motor learning and neuroplasticity is induced or facilitated by robot assistance is rather scarce. However, it has been suggested that robot assistance may promote motivation, because motor performance during training can be better than without assistance $(34,61)$. Clinically, robotassisted therapy is effective after acute and chronic stroke (38), although not more so than intensity-matched physical therapy when a human therapist in part assists, in part motivates the patient's own movements (9). Analysis of biomechanical aspects of motor recovery, suggest that motor learning (i.e., neuroplasticity) and not motor adaptation characterizes motor recovery after robot-assisted therapy, although this is not direct evidence gathered using neuroimaging (62). Robot-assisted therapy generally includes many thousands of repetitive movements over 16 months and this total intensity is required for neuroplasticity in animal models (63). Unfortunately, this intensity is rarely or never matched in other well studied therapies such as CIMT or functional electrical stimulation (FES) or indeed in usual care - the evidence for neuroplasticity following CIMT and FES is preliminary and out of the scope of this perspective [see review by (33)].

In summary, performing motor tasks with robot-mediated assistance can modulate neural activity compared to un-assisted or active voluntary movements in healthy subjects and stroke patients, although whether the patterns of modulation are similar in health and disease remains to be compared. Strong direct evidence for neuroplasticity following robot-assisted therapy is currently lacking and future work is required to identify which type of assistance is optimal for inducing neuroplasticity and thus reducing motor impairment.

\section{ELEMENT 2: ROBOTIC PERTURBATION}

Perturbing a movement, for example by applying an external force, renders it more difficult to perform. Increased difficulty adds to the intensity of training and could serve as a stronger learning stimulus; on the other hand perturbations that are too large may hinder the learning process. Several paradigms using robotic devices have been used to investigate the neuroplasticity that occurs when healthy subjects have learned to "adapt" to a perturbation during reaching or tracking movements of the hand (upper limb) or ankle (lower limb).

\section{EVIDENCE FOR NEUROPLASTICITY: UPPER LIMB}

One common paradigm incorporates adaptation to robot-induced force fields which physically perturb ongoing arm movement (64). Changes in cortico-striatal neural activation, cortical excitability along with short interval intracortical inhibition and facilitation have been demonstrated during such adaptation processes [e.g., $(65,66)$; Figure 2].

Perturbation stimulates the healthy motor system to adapt, that is, to counteract the external force and can involve adaptation of predictive "feedforward anticipatory" movement or force production (an "internal model") and adaptation of reactive "feedback" adjustments of limb movement in response to the perturbation. Adaptation is a fast process that can be distinguished from learning by repeating the same unperturbed movement over and over again (67). Whereas the latter depends on activity (use)-dependent neuroplasticity, cerebellar error-based learning mechanisms may account for adaptation of reaching during force-field perturbations (68). A shift of activation from cortico-striatal to corticocerebellar networks occurs while adapting to an external force field and this is associated with changes in effective connectivity amongst cortical regions in healthy humans $(66,69)$. Shifts in neural network activation during motor tasks persist following rest periods after force-field motor adaptation possibly indicating a "motor memory" consolidation process [i.e., neuroplasticity; (70)]. Persistent memory of motor responses is best achieved by combining error-based adaptation and use-dependent plasticity (67). Whether, this combination of healthy motor learning mechanisms is active during recovery from brain injury remains to be demonstrated. Preliminary studies however, suggest that incorporating error augmentation may be a beneficial strategy for upper limb therapy in chronic stroke patients $(71,72)$.

In healthy subjects, cortical excitability of a brain region can be modulated by applying unilateral anodal tDCS during motor adaptation (Figure 3A). Cerebellar anodal tDCS during armreaching adaptation to visuomotor rotation results in a faster rate of adaptation (73), whereas retention of the "offline motor memory" of adapted behavior is enhanced by anodal stimulation of the primary motor cortex [visuomotor rotation - (73); robot-induced force field - (74); Figures 3B-D].

Recent work using in vitro motor cortex brain slices has suggested that tDCS interacts with coincident low frequency stimulation (in possible analogy to afferent activity accompanying movement during human motor adaptation) to increase BDNF secretion and TrkB activation (75). Both of these induced molecular changes are stimulation(activity)-dependent and characteristic of synaptic neuroplasticity (18). In order to augment neuroplasticity during robot perturbation training in clinical populations in this way, future effort is required to determine the optimal selection of the stimulated brain region (cerebellar vs. cerebral), site of tDCS related to location of injury (ipsilesional vs. contralesional), type of tDCS (anodal vs. cathodal), and the type of robot-mediated therapy (unilateral vs. bilateral). Indeed, studies using single hemisphere tDCS and robot-mediated bilateral assistive therapy in stroke patients did not demonstrate clinically significant effects on motor recovery (76). Repetitive TMS is a similar technique to tDCS in the sense of modulating cortical excitability, however currently it has not been used either with robot-mediated motor adaptation in healthy subjects or with robot-assisted therapy in clinical populations.

\section{EVIDENCE FOR NEUROPLASTICITY: LOWER LIMB}

The concept of perturbation has also been applied to the lower limb in gait training. Short-term motor adaptation can occur in healthy subjects during walking when one limb operates in a force-field environment and the behavioral adaptation is associated with changes in cortical excitability (77). Indeed, when tDCS 

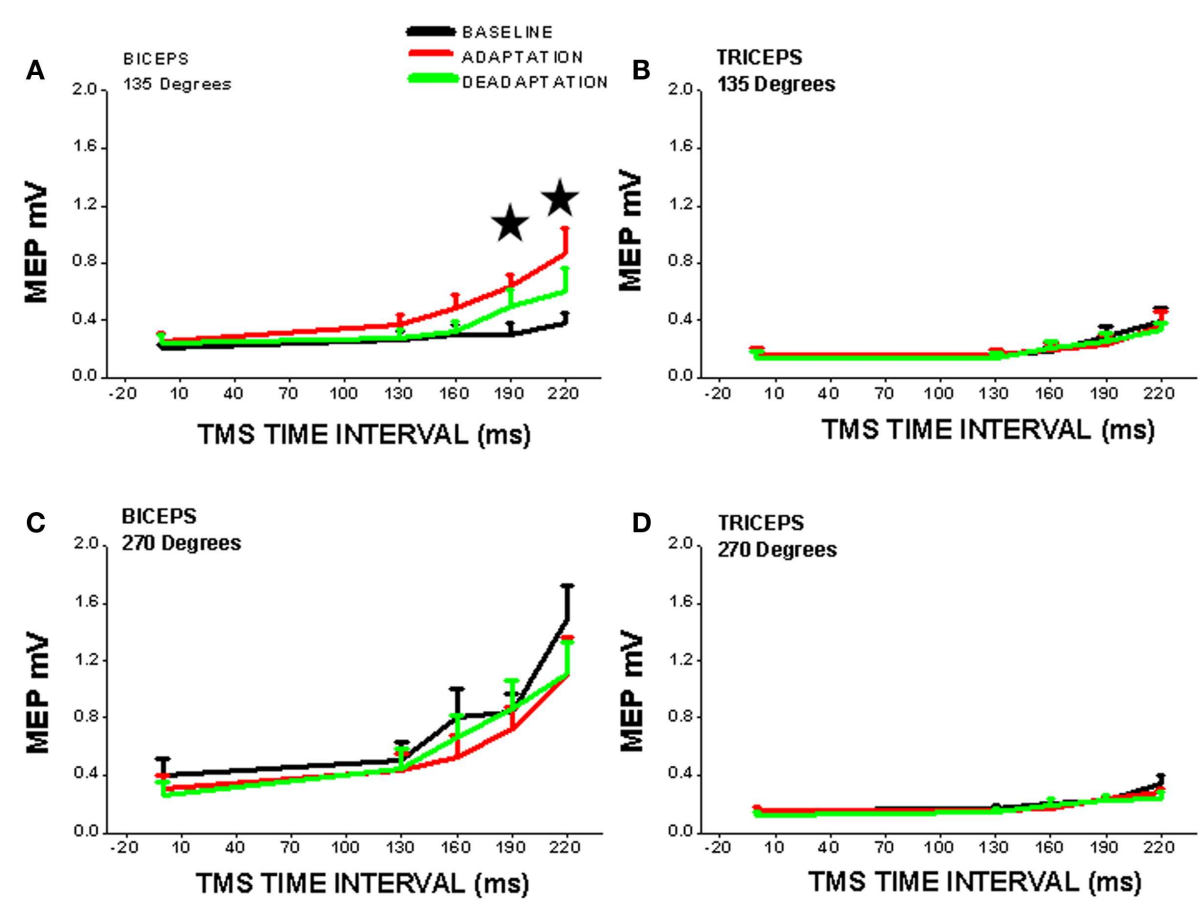

FIGURE 2 | Cortical excitability of contralateral motor cortex is significantly increased (*, vs. BASELINE condition) during robot-mediated clockwise force field perturbation adaptation in healthy subjects (ADAPTATION condition cf. BASELINE and DEADAPTATION conditions). TMS was used to measure cortical excitability during the movement preparation period before reaching (TMS time interval is time after visual signal to start reach and is set at $x=0$ on $x$-axis) to two different directions $\left[(\mathbf{A}, \mathbf{B})=135^{\circ}\right.$, away from

the body; $(\mathbf{C}, \mathbf{D})=270^{\circ}$, toward the chest] and for two different upper limb muscles $[(\mathbf{A}, \mathbf{C})=$ biceps; $(\mathbf{B}, \mathbf{D})=$ triceps]. Note that cortical excitability is only increased for one muscle (biceps) in one direction of perturbed reaching $\left(135^{\circ}\right)$, so cortical neuroplasticity is thus muscle- and direction-specific. The increase in cortical excitability precedes reaching movement and suggests that there is a change in the "internal model" of the biceps muscle within the cortex [from Ref. (65) with permission]. is applied to cerebellum in healthy subjects, the rate of motor adaptation to split-belt treadmill walking can be increased or decreased depending on the modality of tDCS [i.e., anodal vs. cathodal respectively; (78)].

In clinical studies involving patients with cerebellar degeneration, predictive feedforward components of motor adaptation were impaired, whereas reactive feedback components were not impaired, when the patients were walking on a split-belt treadmill with the two belts - one for each leg - running at different velocities (79). On the other hand, stroke patients with cerebral damage could adapt in a similar fashion to healthy control subjects, when performing the same split-belt walking paradigm (80). These differential findings in clinical populations suggest that the cerebellum may be more important than the cerebral cortex in perturbation adaptation in the lower limb. Lasting improvement remains to be demonstrated in large clinical studies but the first trials suggest that gait asymmetry in chronic stroke can be ameliorated by split-belt walking training (81). However the long-term neuroplastic changes underlying the adapted behavior in both healthy subjects and clinical groups are unknown, but deserve future investigation.

In summary, perturbation-based robot-mediated therapy following neurological injury has not received the attention that assistive robot-mediated therapy has and there is a lack of direct comparative evidence to suggest one is better than the other currently. Further, while there is some evidence to suggest that modulation of motor and sensory neural circuits occurs (82) during motor adaptation in health and disease, caution should be kept in mind when translating evidence from learning/adaptation in healthy subjects to stroke compared to cerebellar degeneration, for example. Nevertheless, future studies using combinations of assistive and perturbation-based motor adaptation (71) with or without adjunct non-invasive brain stimulation may be worthwhile clinically and elucidate the impact of robot-mediated perturbation on neuroplasticity per se.

\section{ELEMENT 3: ADDING VIRTUAL REALITY TO ROBOT-MEDIATED THERAPY}

Virtual reality (VR) has been combined with a robotic training device in gait training after stroke and can significantly augment gait improvements more than robot therapy alone (83). The impact of VR on robot-induced gait improvements after stroke is manifest as increases in force and power via improvement of ankle motor control (84). If used appropriately, VR can represent to the stroke patient certain bio-signals related to gait performance such as heart rate or force/torques at lower limb joints and thus stimulate conscious control of precision movement (85).

Unfortunately a significant number of stroke survivors often see little progress in their training because improvement is slow and post-stroke depression may devalue reward. Several rewarding 


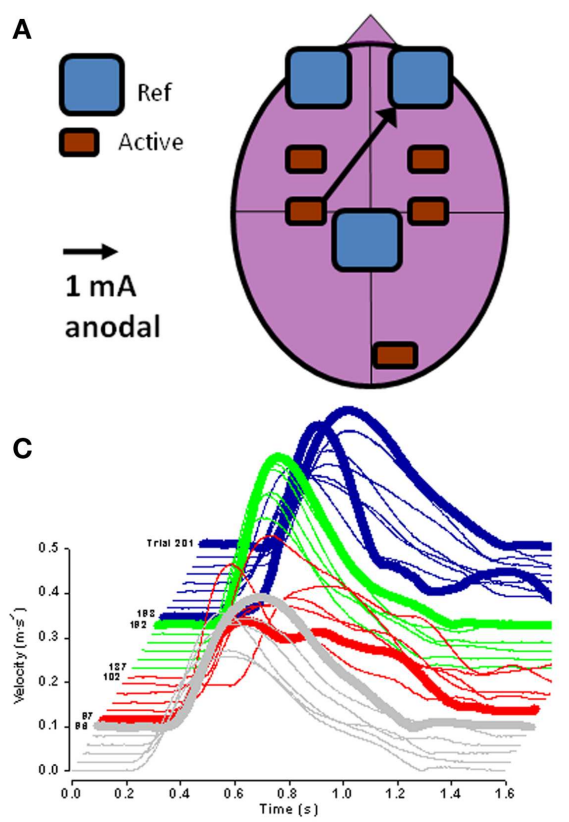

FIGURE 3 | Unilateral anodal tDCS (black arrow with the cathodal electrode applied supraorbitally; (A) was applied to contralateral motor cortex during force-field adaptation in order to augment ongoing neuroplastic changes in cortical neurophysiology (see Figure 2). Interestingly, online tDCS stimulation did not change the reduction of movement error or
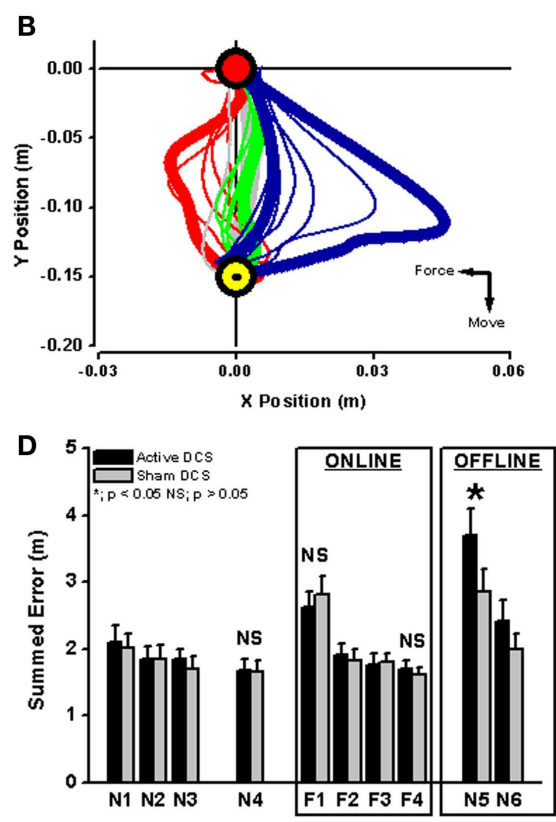

recovery of velocity during motor adaptation [red lines in $(\mathbf{B}, \mathbf{C})$ ], but did result in a significant increase $\left(^{*}\right)$ in offline movement error once tDCS and the robot-induced force field were both switched off (blue lines in (C,D); black bars in (D). Reaching blocks N1-N4 and N5-N6 are without force field and reaching blocks F1-F4 are with force field perturbation. From (74) with permission) features can be built into training robots, such as those providing immediate feedback about movement errors as well as delayed rewards, for example by collecting points or virtual money. VR can provide an excellent framework for reward presentation. Reward regions in the brain have been proposed to contribute to motor skill learning in an animal model via dopaminergic pathways (86). Recent studies in healthy humans have suggested that primary motor cortex, which participates in motor learning, also responds to rewarding of successful behavior - increased reward was correlated with greater paired-pulse inhibition using TMS (87). Furthermore, motor skill learning when performed in positive-reward conditions led to a prolonged long-term retention of a motor memory, whereas neutral or punishment-related skill learning did not (88). Hence, any strategy that enhances reward signals for correct movement sequences via VR or other additional technologies during robot-mediated therapy may have a clinical benefit. In summary, adding VR to robot-mediated therapy remains to be explored both in terms of neuroplasticity and clinical application. Additionally, the type of visual stimuli used in VR-robot environments requires further investigation.

\section{ELEMENT 4: INTERFACING THE BRAIN WITH A ROBOTIC DEVICE}

The idea of using a combination of a BMI and robots for rehabilitation has been explored in several recent studies and is justified by the absence of rehabilitation therapies for paralyzed and severely impaired stroke patients. These patients cannot benefit from existing therapy since residual movement ability is generally necessary.
Rehabilitation robotic devices hold the potential to bridge the gap between the intention to move (i.e., in the CNS) and actual movement of an orthosis or robot device without the need of a limb (89-91). In this section, we deal with neurological patients only, as the fundamental development of BMI systems is outside the perspective scope. However, feasibility studies on healthy subjects are often required initially to investigate synthetic and neurophysiological artifacts when linking the brain of the BMI-user and movements of a limb or robot (40).

Stroke patients can acquire control over a hand orthosis (opening/closing of the hand) by volitionally modulating sensorimotor frequency-dependent rhythms in the lesioned hemisphere. While most of the patients were able to learn controlling the orthosis via the BMI, clinical scales used to measure hand function showed no improvement after training (92). In another study, two groups of sub-acute stroke patients who received either standardized robotic training or BMI-driven robot training demonstrated the ability to improve performance by using motor imagery in the ipsilesional motor cortex (93). A single case study reported recovery of a severely affected chronic stroke patient using a combination of BMI-robot therapy and physiotherapy (94). Functional and anatomical neural correlates of functional clinical outcome measures of recovery were evaluated in a multimodal imaging approach, whereby increased lateralization of neural activity occurred in the ipsilesional hemisphere and white matter reorganization occurred in the ipsilesional corticospinal tract (94). The effectiveness of brain-robot interfaces in stroke rehabilitation may be improved by "closing the feedback loop," whereby 
haptic feedback enables ipsilesional sensorimotor loops to be reactivated (95). Interestingly, the contralesional hemisphere can also be activated during attempted reaching tasks in severely affected chronic stroke patients (96). This suggests that several brain regions have the capability to interact with a robot-effector and the injured brain region can be "bypassed" (97). This would be a good example of how robot based therapeutic design can be built on an understanding of principles of neuroplasticity and the functional connectivity between several brain regions during recovery from stroke. For example, a recent study demonstrated that the motor imagery component of brain-robot interface training can augment changes in functional connectivity in chronic stroke patients beyond that induced solely by robot-assisted therapy (98). Lastly, a very recent controlled study proved the efficacy of a rehabilitation paradigm using BMI and behavioral physiotherapy in chronic stroke patients, closing the loop between brain signals related to movement intention and that same movement via a BMI controlled robotic orthosis (99). In this double-blind feasibility study, 32 chronic stroke severely paralyzed patients (without residual finger extension) received 18 training sessions. One group received contingent BMI-training: ipsilesional corticaldesynchronization was linked to movements of a robotic orthosis fixed to the paralyzed limb. The control group (sham) received the same training, but the movements of the orthotic device were randomized and independent of cortical desynchronization. Both groups received identical behavioral physiotherapy after every BMI-session. The experimental group showed a significant improvement in Fugl-Meyer upper limb motor scores, BMI control, increased muscle activity, and control in the paralyzed hand and arm and lateralization of brain activation toward the ipsilesional hemisphere when compared to the control group.

In summary, this rapidly expanding field is yielding significant methodological steps forward in the design of upper and lower limb rehabilitation using BMI with robots or other hybrid approaches such as acquired self-control of brain activity (12, $50,100,101)$. The neurophysiological mechanisms, measured for example with TMS, underpinning motor improvement; the role of neuroplasticity, and the clinical value of these combined therapy approaches remain to be fully explored.

\section{CRITICAL SUMMARY OF THE POTENTIAL FOR ROBOT-INDUCED NEUROPLASTICITY IN NEUROLOGICAL RECOVERY FROM INJURY}

The use of robot-mediated therapy for augmenting recovery from neurological injury is now becoming more attractive as evidence for cost effectiveness of robot-mediated therapy is becoming stronger $(9,102)$. As robot device use increases, it is likely that an increasing range of therapy strategies will be designed [see Ref. (35) for examples]. The incorporation of neuroimaging and monitoring of neurophysiology alongside robot-mediated therapy is in its infancy in comparison (one example of kinematic analysis is shown in Figure 4). Nevertheless, there is some suggestion that

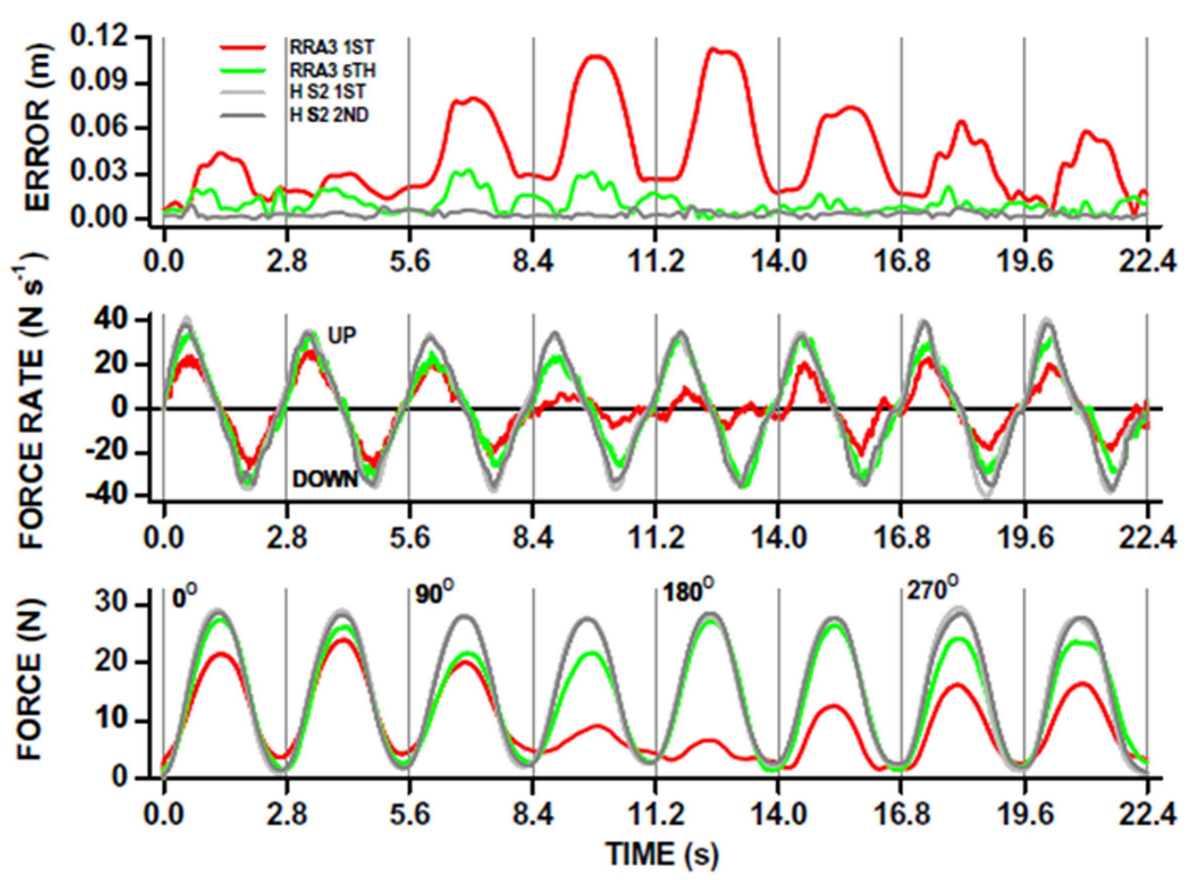

FIGURE 4 | Robot-mediated perturbations can be used to evaluate acute stroke patient motor performance in a "holding" task. The patient is instructed to hold the joystick in the middle of a computer screen and the robot exerts "pulling" forces to the joystick (see also Figure 1D). This acute stroke patient undertook 20, $1 \mathrm{~h}$ therapy sessions, each including $\sim 1000$ robot-assisted reaches to peripheral targets on a computer screen in different directions. The ability to hold the joystick in a central position whilst the robot applied "pulling" forces in different directions, was measured before (red traces; RRA3 first) and after (green traces; RRA3 fifth) the robot-assisted therapy program. The overall $x-y$ position error (top panel) was significantly reduced after robot-assisted therapy toward that measured in healthy subjects (gray traces; HS2 first and second). Note that position holding performance was direction-specific in this patient. The kinematic improvement in position holding was the result of increases in kinetic force production (bottom panel) and also the rate of force production (UP) and relaxation (DOWN; middle panel) toward that of healthy subjects. From Ref. (117) with permission. 
changes in neural activation and functional connectivity can be associated with robot-mediated movement and therapy.

\section{PARADOXES TO RESOLVE IN ROBOT-INDUCED NEUROPLASTICITY}

However, critically assessing the evidence for neuroplasticity induced by robot use has highlighted several large gaps in our knowledge and some paradoxes that should be addressed (Table 1).

The first paradox is that whilst robot-assisted therapy is the most frequently robot-based intervention for neurological injury, we know little about the neural correlates of this type of movement $(41,42)$ and even less on whether an assistance control strategy induces neuroplasticity per se $(42,43,48,49)$. It could be argued that assisting movements (i.e., similar to passively moving the limb) might require less neural activation than actively engaging with a voluntary effort to move a limb, even when it is not possible to move it. Certainly, passive movement elicits a lower neural activation "intensity" and altered regional pattern compared to active movement or motor imagery of the same type of task in stroke patients; note this was not the case in healthy subjects performing the same task and thus translating healthy to neurological concepts warrants caution (103).

The second paradox is that whilst we know a significant amount about the neural correlates of motor learning and neuroplasticity induced by robot-mediated perturbations in healthy subjects, this type of robot usage is rarely used in neurological therapy (71). The neuroplasticity in response to adaptation to robot perturbations of movement can be substantial and widespread. Importantly, recently this type of neuroplasticity induced by motor adaptation was demonstrated to be long lasting even at the single neuron level (104). Future work is required to assess whether robot perturbation type therapy induces neuroplastic changes which correlate with clinical outcome.

The third paradox is that whilst cortico-striatal neural activation is modulated during and after robot perturbing motor adaptation in healthy subjects (66) and reward circuits may be involved in motor skill learning $(86,88)$, the impact of motivation/reward on neuroplasticity during robot-based motor adaptation is not known in either healthy subjects or in neurological patients. This might be important to consider, because there is a growing development of autonomous control of robot-related therapy characteristics (e.g., level of force used; type of assistance and so on) as home-based robot therapy becomes more probable (105-109). Increasingly, the human-robot interaction will require bi-directional input in terms of the patient being able to achieve goals/rewards to maintain high adherence to therapy whilst using the robot on the one hand and the robot being driven optimally by patient performance on the other.

The fourth paradox involves the interaction of brain and robot without the need for an actuating human limb. Using brain signals to drive a robot device directly to undertake everyday tasks and to induce motor rehabilitation is feasible following severe stroke (99, $110,111)$. The changes that occur in neuronal cell tuning properties and firing co-variance, spike timing across neural networks, and spectral changes during the period of learning how to drive the robot by thought or movement intention alone, suggest that
Table 1 | Levels of evidence for neuroplasticity in robot-assisted therapy and robot motor learning employing various elements.

Robot-assisted therapy Robot motor learning

\begin{tabular}{lll}
\hline Assistance & Low & Low \\
Perturbation & Low & High \\
Reward & Low & Low \\
Brain-machine interface & Low & Low \\
\hline
\end{tabular}

neuroplasticity occurs (112). However, it could be that there is a substantial change in neural output over a prolonged time "practicing" the specific task such that the same neuron groups become resistant to learning other new tasks (or their tuned responses that drive the robot task in the first instance suffer from interference). Learning different types of robot-mediated motor perturbations certainly demonstrate patterns of interference which degrade performance and rather rapidly so $(113,114)$; thus BMI induced re-organization might inhibit/interfere with learning future new tasks if the re-organization becomes too "entrenched" in neural circuits involved in movement. Long-term studies of BMI use to study possible neuroplastic changes are required to answer these questions.

\section{CONCLUSION}

There are several large gaps in our knowledge on the neural correlates of effective robot-mediated therapy (Table 1 ). The rapid advances in robot design, but more importantly neuroimaging techniques compatible with robotics (e.g., Figure 1) will catalyze the next steps in understanding the role of robot-mediated neuroplasticity in successful recovery from brain injury.

Robots are more than aids for or simply replacements of therapists to deliver movement therapy. From a technical perspective, robots can be excellent research tools, because they provide ways to standardize rehabilitative training, to precisely monitor recovery of motor function in patients [Figure 4; $(62,115-117)$ ] and to control protocols for subjective human influence. The concepts described in this perspective suggest future work for developing training methods grounded in neurophysiological principles that can be delivered by robotic devices to optimally stimulate neuroplastic processes and learning in the CNS. Most likely no single concept will be the single solution for all patients. Rather combinations will provide a highly individualized training that is delivered in a repetitive and standardized fashion, for example tDCS and robot-mediated therapy (63). This will produce robotbased assessment measures that are comparable across patients with different motor disorders or at different time points of their lifespan (e.g., childhood vs. adult; (118)) in the clinic and at home (119).

\section{ACKNOWLEDGMENTS}

Funded in part by the EU Commission through COST Action "European Network on Robotics for Rehabilitation" (TD1006). Niels Birbaumer is supported by the DFG (Deutsche Forschungsgemeinschaft) and the Bundesministerium für Bildung und Forschung (BMBF): Bernstein Center Tübingen-Freiburg (Nr. 01GQ0831). 


\section{REFERENCES}

1. Olesen J, Gustavsson A, Svensson M, Wittchen HU, Jönsson B, European Brain Council. The economic cost of brain disorders in Europe. Eur J Neurol (2012) 19:155-62. doi:10.1111/j.1468-1331.2011.03590.x

2. Cramer SC, Nudo RJ. Brain Repair after Stroke. Cambridge: Cambridge University Press (2010).

3. Cramer SC, Sur M, Dobkin BH, O’Brien C, Sanger TD, Trojanowski JQ, et al. Harnessing neuroplasticity for clinical applications. Brain (2011) 134:1591-609. doi:10.1093/brain/awr039

4. Dietz V. Neuronal plasticity after a human spinal cord injury: positive and negative effects. Exp Neurol (2012) 235:110-5. doi:10.1016/j.expneurol.2011. 04.007

5. Chollet F, Tardy J, Albucher JF, Thalamas C, Berard E, Lamy C, et al. Fluoxetine for motor recovery after acute ischaemic stroke (FLAME): a randomised placebo-controlled trial. Lancet Neurol (2011) 10:123-30. doi:10.1016/S14744422(10)70314-8

6. Harkema S, Gerasimenko Y, Hodes J, Burdick J, Angeli C, Chen Y, et al. Effect of epidural stimulation of the lumbosacral spinal cord on voluntary movement, standing, and assisted stepping after motor complete paraplegia: a case study. Lancet (2011) 377:1938-47. doi:10.1016/S0140-6736(11)60547-3

7. Hummel FC, Cohen LG. Non-invasive brain stimulation: a new strategy to improve neurorehabilitation after stroke? Lancet Neurol (2006) 5:708-12. doi:10.1016/S1474-4422(06)70525-7

8. Langhorne P, Bernhardt J, Kwakkel G. Stroke rehabilitation. Lancet (2011) 377:1693-702. doi:10.1016/S0140-6736(11)60325-5

9. Lo AC, Guarino PD, Richards LG, Haselkorn JK, Wittenberg GF, Federman DG, et al. Robot-assisted therapy for long-term upper-limb impairment after stroke. N Engl J Med (2010) 362:1772-83. doi:10.1056/NEJMoa0911341

10. Mehrholz J, Pohl M. Electromechanical-assisted gait training after stroke: a systematic review comparing end-effector and exoskeleton devices. J Rehabil Med (2012) 44:193-9. doi:10.2340/16501977-0943

11. Musienko P, Heutschi J, Friedli L, den Brand RV, Courtine G. Multi-system neurorehabilitative strategies to restore motor functions following severe spinal cord injury. Exp Neurol (2012) 235:100-9. doi:10.1016/j.expneurol.2011.08. 025

12. Silvoni S, Ramos-Murguialday A, Cavinato M, Volpato C, Cisotto G, Turolla A, et al. Brain-computer interface in stroke: a review of progress. Clin EEG Neurosci (2011) 42:245-52. doi:10.1177/155005941104200410

13. Wolf SL, Winstein CJ, Miller JP, Taub E, Uswatte G, Morris D, et al. Effect of constraint-induced movement therapy on upper extremity function 3 to 9 months after stroke: the EXCITE randomized clinical trial. JAMA (2006) 296:2095-104. doi:10.1001/jama.296.17.2095

14. Dimyan MA, Cohen LG. Neuroplasticity in the context of motor rehabilitation after stroke. Nat Rev Neurol (2011) 7:76-85. doi:10.1038/nrneurol.2010.200

15. Shadmehr R, Smith MA, Krakauer JW. Error correction, sensory prediction, and adaptation in motor control. Annu Rev Neurosci (2010) 33:89-108. doi:10.1146/annurev-neuro-060909-153135

16. Carmichael ST. Brain excitability in stroke: the Yin and Yang of stroke progression. Arch Neurol (2012) 69:161-7. doi:10.1001/archneurol.2011.1175

17. Caroni P, Donato F, Muller D. Structural plasticity upon learning: regulation and functions. Nat Rev Neurosci (2012) 13:478-90. doi:10.1038/nrn3258

18. Cooke SF, Bliss TV. Plasticity in the human central nervous system. Brain (2006) 129:1659-73. doi:10.1093/brain/awl082

19. Clapp WC, Hamm JP, Kirk IJ, Teyler TJ. Translating long-term potentiation from animals to humans: a novel method for noninvasive assessment of cortical plasticity. Biol Pyschiatry (2012) 71:496-502. doi:10.1016/j.biopsych.2011. 08.021

20. Verhoog MB, Goriounova NA, Obermayer J, Stroeder J, Hjorth JJJ, Test-Silva $\mathrm{G}$, et al. Mechanism underlying the rules for associative plasticity at adult human neocortical synapses. J Neurosci (2013) 33:17197-208. doi:10.1523/ JNEUROSCI.3158-13.2013

21. Nitsche MA, Mueller-Dahlhaus F, Paulus W, Ziemann U. The pharmacology of neuroplasticity induced by non-invasive stimulation: building models for the clinical use of CNS active drugs. J Physiol (2012) 590:4641-62. doi:10.1113/jphysiol.2012.232975

22. Sehm B, Kipping J, Schafer A, Villringer A, Ragert P. A comparison between uni- and bi-lateral tDCS effects on functional connectivity of the human motor cortex. Front Hum Neurosci (2013) 7:183. doi:10.3389/fnhum.2013.00183
23. Polenia R, Paulus W, Antal A, Nitsche MA. Introducing graph theory to track for neuroplastic alterations in the resting human brain: a transcranial direct current stimulation study. Neuroimage (2011) 54:2287-96. doi:10.1016/ j.neuroimage.2010.09.085

24. Polenia R, Paulus W, Nitsche MA. Modulating cortico-striatal and thalamocortical functional connectivity with transcranial direct current stimulation. Hum Brain Mapp (2012) 33:2499-508. doi:10.1002/hbm.21380

25. Dietz V, Fouad K. Restoration of sensorimotor functions after spinal cord injury. Brain (2013). doi:10.1093/brain/awt262

26. Fouad K, Tetzlaff W. Rehabilitative training and plasticity following spinal cord injury. Exp Neurol (2012) 235:91-9. doi:10.1016/j.expneurol.2011. 02.009

27. Grefkes C, Fink GR. Reorganization of cerebral networks after stroke: new insights from neuroimaging with connectivity approaches. Brain (2011) 134:1264-76. doi:10.1093/brain/awr033

28. Kokotilo KJ, Eng JJ, Curt A. Reorganization and preservation of motor control of the brain in spinal cord injury: a systematic review. J Neurotrauma (2009) 26:2113-26. doi:10.1089/neu.2008.0688

29. Langhorne P, Coupar F, Pollock A. Motor recovery after stroke: a systematic review. Lancet Neurol (2009) 8:741-54. doi:10.1016/S14744422(09)70150-4

30. Nishimura Y, Isa T. Cortical and subcortical compensatory mechanisms after spinal cord injury in monkeys. Exp Neurol (2012) 235:152-61. doi:10.1016/j. expneurol.2011.08.013

31. Rossini PM, Calautti C, Pauri F, Baron JC. Post-stroke plastic reorganisation in the adult brain. Lancet Neurol (2003) 2:493-502. doi:10.1016/S1474-4422(03) 00485-X

32. Stinear C. Prediction of recovery of motor function after stroke. Lancet Neurol (2010) 9:1228-32. doi:10.1016/S1474-4422(10)70247-7

33. Takeuchi N, Izumi S-I. Rehabilitation with post-stroke motor recovery: a review with a focus on neural plasticity. Stroke Res Treat (2013) 2013:128641. doi:10.1155/2013/128641

34. Krakauer JW, Mazzoni P. Human sensorimotor learning: adaptation, skill, and beyond. Curr Opin Neurobiol (2011) 21:636-44. doi:10.1016/j.conb.2011.06. 012

35. Marchal-Crespo L, Reinkensmeyer DJ. Review of control strategies for robotic movement training after neurologic injury. J Neuroeng Rehabil (2009) 6:20. doi:10.1186/1743-0003-6-20

36. Sing GC, Joiner WM, Nanayakkara T, Brayanov JB, Smith MA. Primitives for motor adaptation reflect correlated neural tuning to position and velocity. Neuron (2009) 64:575-89. doi:10.1016/j.neuron.2009.10.001

37. Colombo R, Sterpi I, Mazzone A, Delconte C, Minuco G, Pisano F. Measuring changes of movement dynamics during robot-aided neurorehabilitation of stroke patients. IEEE Trans Neural Syst Rehabil Eng (2010) 18:75-85. doi:10.1109/TNSRE.2009.2028831

38. Volpe BT, Huerta PT, Zipse JL, Rykman A, Edwards D, Dipietro L, et al. Robotic devices as therapeutic and diagnostic tools for stroke recovery. Arch Neurol (2009) 66:1086-90. doi:10.1001/archneurol.2009.182

39. Matari MJ, Eriksson J, Feil-Seifer DJ, Winstein CJ. Socially assistive robotics for post-stroke rehabilitation. J Neuroeng Rehabil (2007) 4:5. doi:10.1186/17430003-4-5

40. Ramos-Murguialday A, Schurholz M, Caggiano V, Wilbruger M, Caria A, Hammer EM, et al. Proprioceptive feedback and brain computer interface (BCI) based neuroprostheses. PLoS One (2012) 7:e47048. doi:10.1371/journal.pone. 0047048

41. Formaggio E, Storti SF, Galazzo IB, Gandolfi M, Geroin C, Smania N, et al. Modulation of event-related desynchronization in robot-assisted hand performance: brain oscillatory changes in active, passive and imagined movements. J Neuroeng Rehabil (2013) 10:24. doi:10.1186/1743-0003-10-24

42. Novakovic V, Sanguineti V. Adaptation to constant-magnitude assistive forces: kinematic and neural correlates. Exp Brain Res (2011) 209:425-36. doi:10.1007/ s00221-011-2573-7

43. Lin CL, Shaw FZ, Young KY, Lin CT, Jung TP. EEG correlates of haptic feedback in a visuomotor tracking task. Neuroimage (2012) 60:2258-73. doi:10.1016/j.neuroimage.2012.02.008

44. Onishi H, Sugawara K, Yamashiro K, Sato D, Suzuki M, Kirimoto H, et al. Neuromagnetic activation following active and passive finger movements. Brain Behav (2013) 3:178-92. doi:10.1002/brb3.126 
45. Weiller C, Juptner M, Fellows S, Rijntjes M, Leonhardt G, Kiebel S, et al. Brain representation of active and passive movements. Neuroimage (1996) 4:105-10. doi:10.1006/nimg.1996.0034

46. Lotze M, Braun C, Birbaumer N, Anders S, Cohen LG. Motor learning elicited by voluntary drive. Brain (2003) 126:866-72. doi:10.1093/brain/awg079

47. Perez MA, Lungholt BKS, Nyborg K, Nielsen JB. Motor skill training induces changes in the excitability of the leg cortical area in healthy humans. Exp Brain Res (2004) 159:197-205. doi:10.1007/s00221-004-1947-5

48. Takahashi CD, Der-Yeghiaian L, Le V, Motiwala RR, Cramer SC. Robot-based hand motor therapy after stroke. Brain (2008) 131:425-37. doi:10.1093/brain/ awm 311

49. Sergi F, Krebs HI, Groissier B, Rykman A, Guglielmelli E, Volpe BT, et al. Predicting efficacy of robot-aided rehabilitation in chronic stroke patients using an MRI-compatible robotic device. Conf Proc IEEE Eng Med Biol Soc (2011) 2011:7470-3.

50. Belda-Lois JM, Mena-del Horno S, Bermejo-Bosch I, Moreno JC, Pons JL, Farina D, et al. Rehabilitation of gait after stroke: a review towards a top-down approach. J Neuroeng Rehabil (2011) 8:66. doi:10.1186/1743-0003-8-66

51. Knikou M. Plasticity of corticospinal neural control after locomotor training in human spinal cord injury. Neural Plast (2012) 2012:254948. doi:10.1155/ $2012 / 254948$

52. Jayaram G, Stagg CJ, Esser P, Kischka U, Stinear J, Johansen-Berg H. Relationships between functional and structural corticospinal tract integrity and walking post stroke. Neurophysiol Clin (2012) 123:2422-8. doi:10.1016/j.clinph. 2012.04.026

53. Freund P, Weiskopf N, Ward NS, Hutton C, Gall A, Ciccarelli O, et al. Disability, atrophy and cortical reorganisation following spinal cord injury. Brain (2011) 134:1610-22. doi:10.1093/brain/awr093

54. Pomeroy VM, Rowe P, Baron J-C, Clark A, Sealy R, Ugbolue UC, et al. The SWIFT Cast trial protocol: a randomized controlled evaluation of the efficacy of an ankle-foot cast on walking recovery early after stroke and the neural-biomechanical correlates of response. Int J Stroke (2011) 7:86-93. doi:10.1111/j.1747-4949.2011.00704.x

55. Luft AR, Macko RF, Forrester LW, Villagra F, Ivey F, Sorkin JD, et al. Treadmill exercise activates subcortical neural networks and improves walking after stroke. Stroke (2008) 39:3341-50. doi:10.1161/STROKEAHA.108.527531

56. Enzinger C, Dawes H, Johansen-Berg H, Wade D, Bogdanovic M, Collett J, et al. Brain activity changes associated with treadmill training after stroke. Stroke (2009) 40:2460-7. doi:10.1161/STROKEAHA.109.550053

57. Forrester LW, Hanley DF, Macko RF. Effects of treadmill exercise on transcranial magnetic stimulation-induced excitability to quadriceps after stroke. Arch Phys Med Rehabil (1996) 87:229-34. doi:10.1016/j.apmr.2005.10.016

58. Winchester P, McColl R, Querry R, Foreman N, Mosby J, Tansey K, et al. Changes in supraspinal activation patterns following robotic locomotor therapy in motor-incomplete spinal cord injury. Neurorehabil Neural Repair (2005) 19:313-24. doi:10.1177/1545968305281515

59. Yen CL, Wang RY, Liao KK, Huang CC, Yang YR. Gait training induced change in corticomotor excitability in patients with chronic stroke. Neurorehabil Neural Repair (2008) 22:22-30. doi:10.1177/1545968307301875

60. Sacco K, Cauda F, D’Agata F, Duca S, Zettin M, Virgilio R, et al. A combined robotic and cognitive training for locomotor rehabilitation: evidences of cerebral functional reorganization in two chronic traumatic brain injured patients. Front Hum Neurosci (2011) 5:146. doi:10.3389/fnhum.2011.00146

61. Reinkensmeyer DJ, Patton JL. Can robots help the learning of skilled actions? Exerc Sport Sci Rev (2009) 37:43-51. doi:10.1097/JES.0b013e3181912108

62. Dipietro L, Krebs HI, Volpe BT, Stein J, Bever C, Mernoff ST, et al. Learning, not adaptation, characterizes stroke motor recovery: evidence from kinematic changes induced by robot-assisted therapy in trained and untrained task in the same workspace. IEEE Trans Neural Syst Rehabil Eng (2012) 20:48-57. doi:10.1109/TNSRE.2011.2175008

63. Krakauer JW, Carmichael ST, Corbett D, Wittenberg GF. Getting neurorehabilitation right: what can be learned from animal models? Neurorehabil Neural Repair (2012) 26:923-31. doi:10.1177/1545968312440745

64. Shadmehr R, Mussa-Ivaldi FA. Adaptive representation of dynamics during learning of a motor task. J Neurosci (1994) 14:3208-24.

65. Hunter T, Sacco P, Turner DL. Changes in excitability of the motor cortex associated with internal model formation during intrinsic visuomotor learning in the upper arm. J Behav Brain Sci (2011) 1:140-52. doi:10.4236/jbbs.2011.13019
66. Krebs HI, Brashers-Krug T, Rauch SL, Savage CR, Hogan N, Rubin RH, et al. Robot-aided functional imaging: application to a motor learning study. Hum Brain Mapp (1998) 6:59-72. doi:10.1002/(SICI) 1097-0193(1998)6:1<59: :AID-HBM5>3.3.CO;2-B

67. Huang VS, Haith A, Mazzoni P, Krakauer JW. Rethinking motor learning and savings in adaptation paradigms: model-free memory for successful actions combines with internal models. Neuron (2011) 70:787-801. doi:10.1016/j. neuron.2011.04.012

68. Rabe K, Livne O, Gizewski ER, Aurich V, Beck A, Timmann D, et al. Adaptation to visuomotor rotation and force field perturbation is correlated to differen brain areas in patients with cerebellar degeneration. J Neurophysiol (2009) 101:1961-71. doi:10.1152/jn.91069.2008

69. Doyon J, Bellec P, Amsel R, Penhune V, Monchi O, Carrier J, et al. Contributions of the basal ganglia and functionally related brain structures to motor learning. Behav Brain Res (2009) 199:61-75. doi:10.1016/j.bbr.2008.11.012

70. Shadmehr R, Holcomb HH. Neural correlates of motor memory consolidation. Science (1997) 277:821-5. doi:10.1126/science.277.5327.821

71. Patton JL, Stoykov ME, Kovic M, Mussa-Ivaldi FA. Evaluation of robotic training forces that either enhance or reduce error in chronic hemiparetic stroke survivors. Exp Brain Res (2006) 168:368-83. doi:10.1007/s00221-0050097-8

72. Abdollahi F, Case Lazarro ED, Listenberger M, Kenyon RV, Kovic M, Bogey RA, et al. Error augmentation enhancing arm recovery in individuals with chronic stroke: a randomized crossover design. Neurorehabil Neural Repair (2013). doi:10.1177/1545968313498649

73. Galea JM, Vazquez A, Pasricha N, de Xivry JJ, Celnik P. Dissociating the roles of the cerebellum and motor cortex during adaptive learning: the motor cortex retains what the cerebellum learns. Cereb Cortex (2011) 21:1761-70. doi:10.1093/cercor/bhq246

74. Hunter T, Sacco P, Nitsche MA, Turner DL. Modulation of internal model formation during force field-induced motor learning by anodal transcranial direct current stimulation of primary motor cortex. J Physiol (2009) 587:2949-61. doi:10.1113/jphysiol.2009.169284

75. Fritsch B, Reis J, Martinowich K, Schambra HM, Ji Y, Cohen LG, et al. Direct current stimulation promotes BDNF-dependent synaptic plasticity: potential implications for motor learning. Neuron (2010) 66:198-204. doi:10.1016/j. neuron.2010.03.035

76. Hesse S, Waldner A, Mehrholz J, Tomelleri C, Pohl M, Werner C. Combined transcranial direct current stimulation and robot-assisted arm training in subacute stroke patients: an exploratory, randomized multicenter trial. Neurorehabil Neural Repair (2011) 25:838-46. doi:10.1177/1545968311413906

77. Barthélemy D, Alain S, Grey MJ, Nielsen JB, Bouyer LJ. Rapid changes in corticospinal excitability during force field adaptation of human walking. Exp Brain Res (2012) 217:99-115. doi:10.1007/s00221-011-2977-4

78. Jayaram G, Tang B, Pallegadda R, Vasudevan EVL, Celnik P, Bastian A. Modulating locomotor adaptation with cerebellar stimulation. J Neurophysiol (2012) 107:2950-7. doi:10.1152/jn.00645.2011

79. Morton SM, Bastian AJ. Cerebellar contributions to locomotor adaptations during split belt treadmill walking. J Neurosci (2006) 26:9107-16. doi:10.1523/ JNEUROSCI.2622-06.2006

80. Reisman DS, Wityk R, Silver K, Bastian AJ. Locomotor adaptation on a split-belt treadmill can improve walking symmetry post-stroke. Brain (2007) 130:1861-72. doi:10.1093/brain/awm035

81. Reisman DS, McLean H, Keller J, Danks KA, Bastian AJ. Repeated split-belt treadmill training improves poststroke step length asymmetry. Neurorehabil Neural Repair (2013) 27:460-8. doi:10.1177/1545968312474118

82. Nasir SM, Darainy M, Ostry DJ. Sensorimotor adaptation changes the neural coding of somatosensory stimuli. J Neurophysiol (2013) 109:2077-85. doi:10. 1152/jn.00719.2012

83. Mirelman A, Bonato P, Deutsch JE. Effects of training with a robot-virtual reality system compared with a robot alone on the gait of individuals after stroke. Stroke (2009) 40:169-74. doi:10.1161/STROKEAHA.108.516328

84. Mirelman A, Patritti BL, Bonato P, Deutsch JE. Effects of virtual reality training on gait biomechanics of individuals post-stroke. Gait Posture (2010) 31:433-7. doi:10.1016/j.gaitpost.2010.01.016

85. Koenig A, Omlin X, Bergmann J, Zimmerli L, Bolliger M, Müller F, et al. Controlling patient participation during robot-assisted gait training. J Neuroeng Rehabil (2011) 8:14. doi:10.1186/1743-0003-8-14 
86. Hosp JA, Pekanovic A, Rioult-Pedotti MS, Luft AR. Dopaminergic projections from midbrain to primary motor cortex mediate motor skill learning. $\mathrm{J} \mathrm{Neu}$ rosci (2011) 31:2481-7. doi:10.1523/JNEUROSCI.5411-10.2011

87. Kapogiannis D, Campion P, Grafman J, Wassermann EM. Reward-related activity in the human motor cortex. Eur J Neurosci (2008) 27:1836-42. doi:10.1111/j.1460-9568.2008.06147.x

88. Abe M, Schambra H, Wassermann EM, Luckenbaugh D, Schweighofer N, Cohen LG. Reward improves long-term retention of a motor memory through induction of offline memory gains. Curr Biol (2011) 21:557-62. doi:10.1016/j. cub.2011.02.030

89. Birbaumer N, Ramos-Murguialday A, Cohen L. Brain-Computer-Interface (BCI) in paralysis. Curr Opin Neurol (2008) 21:634-8. doi:10.1097/WCO. 0b013e328315ee2d

90. Daly JJ, Wolpaw JR. Brain-computer interfaces in neurological rehabilitation. Lancet Neurol (2008) 7:1032-43. doi:10.1016/S1474-4422(08)70223-0

91. Dobkin BH. Brain-computer interface technology as a tool to augment plasticity and outcomes for neurological rehabilitation. J Physiol (2007) 579:637-42. doi:10.1113/jphysiol.2006.123067

92. Buch E, Weber C, Cohen LG, Braun C, Dimyan MA, Ard T, et al. Think to move: a neuromagnetic brain-computer interface (BCI) system for chronic stroke. Stroke (2008) 39:910-7. doi:10.1161/STROKEAHA.107.505313

93. Ang KK, Guan C, Chua KS, Ang BT, Kuah C, Wang C, et al. Clinical study of neurorehabilitation in stroke using EEG-based motor imagery brain-computer interface with robotic feedback. Conf Proc IEEE Eng Med Biol Soc (2010) 2010:5549-52. doi:10.1109/IEMBS.2010.5626782

94. Caria A, Weber C, Brötz D, Ramos A, Ticini LF, Gharabaghi A, et al. Chronic stroke recovery after combined BCI training and physiotherapy: a case report. Psychophysiology (2011) 48:578-82. doi:10.1111/j.1469-8986.2010.01117.x

95. Gomez-Rodrigues M, Peters J, Hill J, Scholkopf B, Gharabaghi A, GrosseWentrup M. Closing the sensorimotor loop: haptic feedback facilitates decoding of motor imagery. J Neural Eng (2011) 8:036005. doi:10.1088/1741-2560/ $8 / 3 / 036005$

96. Antelis JM, Montesano L, Ramos-Murguialday A, Birbaumer N, Minguez J. Continuous decoding of intention to move from contralesional hemisphere brain oscillations in severely affected chronic stroke patients. Conf Proc IEEE Eng Med Biol Soc (2012) 2012:4099-103.

97. Ganguly K, Secundo L, Ranade G, Orsborn A, Chang EF, Dimitrov DF, et al. Cortical representation of ipsilateral arm movements in monkey and man. J Neurosci (2009) 29:12948-56. doi:10.1523/JNEUROSCI.2471-09.2009

98. Varkuti B, Guan C, Pan Y, Phua KS, Ang KK, Kuah CW, et al. Resting state changes in functional connectivity correlate with movement recovery for BCI and robot-assisted upper-extremity training after stroke. Neurorehabil Neural Repair (2013) 27:53-62. doi:10.1177/1545968312445910

99. Ramos-Murguialday A, Broetz D, Rea M, Lacer L, Yilmaz O, Brasil FL, et al. Brain-machine-interface in chronic stroke rehabilitation: a controlled study. Ann Neurol (2013) 74:100-8. doi:10.1002/ana.23879

100. Caria A, Sitaram R, Birbaumer N. Real-time fMRI: a tool for local brain regulation. Neuroscientist (2012) 18:487-501. doi:10.1177/1073858411407205

101. Minati L, Nigri A, Rosazza C, Bruzzone MG. Thoughts turned into highlevel commands: proof-of-concept study of a vision-guided robot arm driven by functional MRI (fMRI) signals. Med Eng Phys (2012) 34:650-8. doi:10.1016/j.medengphy.2012.02.004

102. Wagner TH, Lo AC, Peduzzi P, Bravata DM, Huang GD, Krebs HI, et al. An economic analysis of robot-assisted therapy for long-term upper-limb impairment after stroke. Stroke (2011) 42:2630-2. doi:10.1161/STROKEAHA.110.606442

103. Szameitat AJ, Shen S, Conforto A, Sterr A. Cortical activation during executed, imagined, observed and passive wrist movements in healthy volunteers and stroke patients. Neuroimage (2012) 62:266-80. doi:10.1016/j.neuroimage. 2012.05.009

104. Richardson AG, Borghi T, Bizzi E. Activity of the same motor cortex neurons during repeated experience with perturbed movement dynamics. J Neurophysiol (2012) 107:3144-54. doi:10.1152/jn.00477.2011

105. Colombo R, Pisano F, Micera S, Mazzone A, Delconte C, Carrozza MC, et al. Assessing mechanisms of recovery during robot-aided neurorehabilitation of the upper limb. Neurorehabil Neural Repair (2008) 22:50-63. doi:10.1177/1545968307303401
106. Colombo R, Sterpi I, Mazzone A, Delconte C, Pisano F. Taking a lesson from patients' recovery strategies to optimize training during robotaided rehabilitation. IEEE Trans Neural Syst Rehabil Eng (2012) 20:276-85. doi:10.1109/TNSRE.2012.2195679

107. Panarese A, Colombo R, Sterpi I, Pisano F, Micera S. Tracking motor improvement at the subtask level during robot-aided neurorehabilitation of stroke patients. Neurorehabil Neural Repair (2012) 26:822-33. doi:10.1177/ 1545968311431966

108. Vergaro E, Casadio M, Squeri V, Giannoni P, Morasso P, Sanguineti V. Selfadaptive robot training of stroke survivors for continuous tracking movements. J Neuroeng Rehabil (2010) 7:13. doi:10.1186/1743-0003-7-13

109. Zimmerli L, Krewer C, Gassert R, Müller F, Riener R, Lünenburger L. Validation of a mechanism to balance exercise difficulty in robot-assisted upper-extremity rehabilitation after stroke. J Neuroeng Rehabil (2012) 9:6. doi:10.1186/1743-0003-9-6

110. Collinger JL, Wodlinger B, Downey JE, Wang W, Tyler-Kabara EC, Weber DJ, et al. High-performance neuroprosthetic control by an individual with tetraplagia. Lancet (2013) 381:557-64. doi:10.106/S0140-6736(12)61816-9

111. Hochberg LR, Bacher D, Jarosiewicz B, Masse NY, Simeral JD, Vogel J, et al. Reach and grasp by people with tetraplegia using a neurally controlled robotic arm. Nature (2012) 485(7398):372-5. doi:10.1038/nature11076

112. Nicolelis MAL, Lebedev MA. Principles of neural ensemble physiology underlying the operation of brain-machine interfaces. Nat Rev Neurosci (2009) 10:530-40. doi:10.1038/nrn2653

113. Cothros N, Wong J, Gribble PL. Distinct haptic cues do not reduce interference when learning to reach in multiple force fields. PLoS One (2008) 3:e1990. doi:10.1371/journal.pone.0001990

114. Howard IS, Ingram JN, Franklin DW, Wolpert DM. Gone in 0.6 seconds: the encoding of motor memories depends on recent sensorimotor states. JNeurosci (2012) 32:12756-68. doi:10.1523/JNEUROSCI.5909-11.2012

115. Bosecker C, Dipietro L, Volpe B, Krebs HI. Kinematic robot-based evaluation scales and clinical counterparts to measure upper limb motor performance in patients with chronic stroke. Neurorehabil Neural Repair (2010) 24:62-9. doi:10.1177/1545968309343214

116. Scott S, Dukelow SP. Potential of robots as next-generation technology for clinical assessment of neurological disorders and upper-limb therapy. J Rehabil Res Dev (2011) 48:335-54. doi:10.1682/JRRD.2010.04.0057

117. Turner DL, Tang X, Winterbotham W, Kmetova M. Recovery of submaximal upper limb force production is correlated with better arm position control and motor impairment early after a stroke. Clin Neurophysiol (2012) 123:183-92. doi:10.1016/j.clinph.2011.06.009

118. Turner DL, Winterbotham W, Kmetova M. Using assistive robotic technology in motor neurorehabilitation after childhood stroke. J Neurol Res (2012) 2:65-8. doi:10.4021/jnr98w

119. Linder SM, Rosenfeldt AN, Reiss A, Buchana S, Sahu K, Bay CR, et al. The home stroke rehabilitation and monitoring system trial: a randomized controlled trial. Int J Stroke (2013) 8:46-53. doi:10.1111/j.1747-4949.2012. 00971.x

Conflict of Interest Statement: The authors declare that the research was conducted in the absence of any commercial or financial relationships that could be construed as a potential conflict of interest.

Received: 10 July 2013; paper pending published: 18 September 2013; accepted: 27 October 2013; published online: 13 November 2013.

Citation: Turner DL, Ramos-Murguialday A, Birbaumer N, Hoffmann U and Luft A (2013) Neurophysiology of robot-mediated training and therapy: a perspective for future use in clinical populations. Front. Neurol. 4:184. doi: 10.3389/fneur.2013.00184 This article was submitted to Neuroprosthetics, a section of the journal Frontiers in Neurology.

Copyright (c) 2013 Turner, Ramos-Murguialday, Birbaumer, Hoffmann and Luft. This is an open-access article distributed under the terms of the Creative Commons Attribution License (CC BY). The use, distribution or reproduction in other forums is permitted, provided the original author(s) or licensor are credited and that the original publication in this journal is cited, in accordance with accepted academic practice. No use, distribution or reproduction is permitted which does not comply with these terms. 